\title{
BISON Software V\&V Plan
}

July 2014

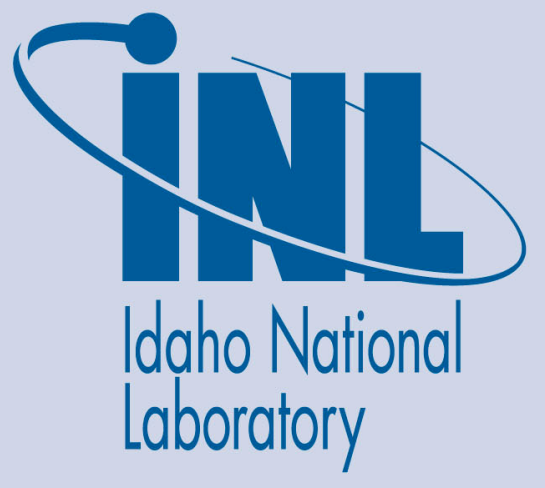

The INL is a U.S. Department of Energy National Laboratory operated by Battelle Energy Alliance 


\section{BISON Software V\&V Plan}

July 2014

Idaho National Laboratory

Fuels Modeling \& Simulation Department Idaho Falls, Idaho 83415

http://www.inl.gov

Prepared for the

U.S. Department of Energy

Office of Nuclear Energy

Under DOE Idaho Operations Office

Contract DE-AC07-05ID14517 


\section{BISON Software V\&V Plan}

Fuels Modeling \& Simulation Department

Idaho National Laboratory

Idaho Falls, ID

July 1, 2014 


\section{Contents}

1 Introduction 3

2 Acronyms 5

3 BISON Description 6

3.1 Tool Description . . . . . . . . . . . . . . . . . . 6

3.2 Code dependencies: Software and Hardware . . . . . . . . . . . . . 7

4 Software Quality Assurance $\quad 9$

5 Verification 10

6 Validation $\quad 12$

6.1 Light Water Reactor Fuel . . . . . . . . . . . . . . . . . . . . . . . . 12

6.1 .1 Collaborations . . . . . . . . . . . . . . . . . . . . 12

6.1 .2 Validation Approach . . . . . . . . . . . . . . . . . . . . 14

6.1 .3 Validation Areas . . . . . . . . . . . . . . . . . . 15

6.1 .4 Current Status . . . . . . . . . . . . . . . . . . . . . 15

6.1 .5 Planned Work . . . . . . . . . . . . . . . . . . . . . . . 16

6.2 TRISO Particle Fuel . . . . . . . . . . . . . . . . . . . . . . . . . . 19

6.2 .1 Code comparisons . . . . . . . . . . . . . . . . . . . . . . 19

6.2 .2 Future work . . . . . . . . . . . . . . . . . . . . 21

6.3 Metallic Fuel . . . . . . . . . . . . . . . . . . . . . . 22

6.3 .1 Collaborations . . . . . . . . . . . . . . . 22

7 Uncertainty Quantification 23

7.1 Accomplished results . . . . . . . . . . . . . . . . . . 23

7.2 Future work . . . . . . . . . . . . . . . . . . . . . 24

8 Supporting Documents $\quad 25$ 


\section{Introduction}

As a component of DOEs Nuclear Energy Advanced Modeling \& Simulation (NEAMS) program, the objective of the Fuels Product Line (FPL) is to deliver an integrated set of mechanistic computational tools for use in nuclear fuel performance analysis and design. A multiscale approach to modeling and simulation has been adopted in developing the FPL toolset in which simulations of fuel performance at the engineering-scale are informed by material property and irradiation performance models developed from meso-scale simulations of microstructure evolution under irradiation, which are themselves enabled by inputs of fundamental materials parameters obtained from atomistic simulations. The tool currently under development for simulations of fuel performance at the engineering-scale is BISON. The tool currently under development for performing meso-scale simulations of microstructure evolution under irradiation, from which material property and irradiation performance models can be derived, is MARMOT. Both BISON and MARMOT are MOOSE-based applications. MOOSE (Multiphysics ObjectOriented Simulation Environment) is a simulation framework that enables rapid development of applications using the finite element method. The MOOSE-BISON-MARMOT suite of tools is sometimes abbreviated as MBM.

Ultimately BISON will be applicable to a wide variety of nuclear fuel forms and reactor applications, including oxide fuels for Light Water Reactors (LWR), oxide and metallic fuels for Sodium Fast Reactors (SFR), TRISO particle fuels for high temperature gas reactors, and even plate-type fuels for research and test reactors. In fact, BISON already has capability to model each of these fuel forms/reactor applications to a greater or lesser degree. Currently, however, the emphasis on the development and implementation of new mechanistic models, demonstration problems, and validation studies have been on oxide fuels under LWR conditions. The basis for this emphasis is two-fold: 1) there is a large, existing fleet of commercial LWRs that could immediately benefit from the improvements in fuel modeling and simulation capabilities provided by BISON, and 2) there is a wealth of existing experimental data and benchmark problems on oxide fuels under LWR conditions with which to assess the multiscale modeling approach undertaken by the NEAMS Fuels Product Line. Demonstrated success of the approach will give confidence in extending BISON into other fuel/reactor systems.

The primary vision for the BISON development team is to deliver a nuclear fuel performance simulation tool that is used to provide a researcher or fuel designer with best estimate calculations of the highly coupled and nonlinear phenomena that govern nuclear fuel behavior. Accurately simulating nuclear fuel behavior is a challenging computational undertaking and verification and validation $(\mathrm{V} \& \mathrm{~V})$ play an important role in realizing this vision. The purpose of this $\mathrm{V} \& \mathrm{~V}$ plan is to express the BISON team's definition of the terms verification and validation, document what we have done regarding $\mathrm{V} \& \mathrm{~V}$, and outline what we plan to do.

The first $\mathrm{V}$ in $\mathrm{V} \& \mathrm{~V}$ is verification, which is the process of determining whether a software implementation of a mathematical model accurately represents that model. By its own definition, 
it is most applicable to individual modules or submodels in an overall system model. With verification showing that each submodel calculates the correct result for a representative set of mathematically-conceived problems, the code can be used with confidence as the validation work begins.

Validation is comparing computed results to experiment measurements. Typically, this data comes from coordinated research efforts that involve power and experimental reactors and postirradiation measurements.

As BISON developers, we feel an obligation to focus on the verification part of the V\&V effort. We have control over the development process and verify each addition to the code independently through regression testing. We feel that validation, on the other hand, is an ongoing effort that must include interaction between developers and users and evolves as more information becomes available (e. g. more measurements from experiments) and as the requirements for an individual user change. In this document, details are provided that document our verification efforts, and examples of simulations that help build a case for validation are shown. 


\section{Acronyms}

$\begin{array}{ll}\text { CRP } & \text { Coordinated Research Project } \\ \text { FGR } & \text { Fission Gas Release } \\ \text { FCRD } & \text { Fuel Cycle Research and Development } \\ \text { FPL } & \text { Fuels Product Line } \\ \text { FUMAC } & \text { Fuel Modeling Under Accident Conditions } \\ \text { FUMEX } & \text { Fuel Modeling at EXtended Burnup } \\ \text { HTGR } & \text { High Temperature Gas Reactor } \\ \text { IAEA } & \text { International Atomic Energy Agency } \\ \text { INL } & \text { Idaho National Laboratory } \\ \text { IPyC } & \text { Inner Pyrolytic Carbon } \\ \text { JFNK } & \text { Jacobian Free Newton Krylov } \\ \text { LWR } & \text { Light Water Reactor } \\ \text { MBM } & \text { MOOSE BISON MARMOT } \\ \text { MOOSE } & \text { Multiphysics Object Oriented Simulation Environment } \\ \text { NEA } & \text { Nuclear Energy Agency } \\ \text { NEAMS } & \text { Nuclear Energy Advanced Modeling and Simulation } \\ \text { OECD } & \text { Organization for Economic Co-operation and Development } \\ \text { PCMI } & \text { Pellet-Cladding Mechanical Interaction } \\ \text { SFR } & \text { Sodium Fast Reactor } \\ \text { SVN } & \text { Subversion } \\ \text { TRISO } & \text { TRistructural ISOtropic } \\ & \end{array}$




\section{BISON Description}

\subsection{Tool Description}

BISON is a modern finite-element based nuclear fuel performance code that has been under development at the Idaho National Laboratory (USA) since 2009 [1]. The code is applicable to both steady and transient fuel behavior and can be used to analyze 1D (spherically symmetric), 2D (axisymmetric and plane strain) or 3D geometries. BISON has been used to investigate a variety of fuel forms including LWR oxide fuel [1], TRISO coated-particle fuel [2] and metallic fuel in rod and plate form [3,4]. The code has also been used to design and to interpret irradiation experiments [5] and investigate novel fuel concepts [6].

BISON is built using the INL Multiphysics Object-Oriented Simulation Environment, or MOOSE [7]. MOOSE is a massively parallel, finite element-based framework to solve systems of coupled non-linear partial differential equations using the Jacobian-Free Newton Krylov (JFNK) method [8]. This enables investigation of computationally large problems, for example a full stack of discrete pellets in a LWR fuel rod, or every rod in a full reactor core. MOOSE supports the use of complex two and three-dimensional meshes and uses implicit time integration, important for the widely varied time scale in nuclear fuel simulation. An object-oriented architecture is employed which greatly minimizes the programming effort required to add new material and behavioral models.

The BISON governing relations currently consist of fully-coupled partial differential equations for energy, species, and momentum conservation. Users can select a subset of these equations (e.g., energy and momentum for thermomechanics analysis) within the input file. The code employs both nonlinear kinematics, which accounts for large deformation, and nonlinear material behavior. A detailed description of the nonlinear kinematics is provided in [1]. For nonlinear plasticity and creep, strains are calculated implicitly utilizing the radial return method; the specific procedure is outlined in [9].

Focusing initially on $\mathrm{UO}_{2}$ fuel, models are included in BISON to describe temperature and burnup dependent thermal properties, solid and gaseous fission product swelling, densification, thermal and irradiation creep, fracture via relocation or smeared cracking, and fission gas production, generation, and release. For TRISO coated-particle fuel, an empirical model is included to compute $\mathrm{CO}$ production, which can be added to released fission gas to affect particle pressure [2].

Recently an improved fission gas release model was implemented in BISON, based on the work of Pastore et al. [10]. While retaining a physics-based description of the relevant mechanisms, the model is characterized by a level of complexity suitable for application to engineeringscale nuclear fuel analysis and consistent with the uncertainties pertaining to key input parameters. The treatment includes the fundamental features of fission gas behavior, among which are gas diffusion and precipitation in fuel grains, growth and coalescence of gas bubbles at grain 
faces, grain growth and grain boundary sweeping effects, thermal, athermal, and transient gas release.

Focusing initially on Zircaloy as a cladding material, models are available for instantaneous plasticity, thermal and irradiation creep, and irradiation growth. The plasticity and creep models can be applied simultaneously, in cases where both phenomena are active.

Gap heat transfer is modeled in the traditional manner with the total conductance across the gap computed as a sum of the gas conductance, the increased conductance due to solid-solid contact, and the conductance due to radiant heat transfer [11]. This model is typically applied between the fuel and cladding, but can also be used to simulate heat transfer between individual pellets, between a pellet and end cap, or between fracture surfaces.

Mechanical contact between materials is implemented through the use of node/face constraints, which prevent nodes on one side of an interface from penetrating faces on the other side of the interface [12]. Finite element contact is notoriously difficult to make efficient and robust in three dimensions and continuous effort is underway to improve the mechanical contact algorithms in BISON.

For LWR fuel, the pressure in the gap and plenum is computed assuming a single cavity volume and using the ideal gas law. The moles of gas, the temperature, and the cavity volume are free to change with time. The moles of gas at any time is computed as the original amount of gas (computed based on original pressure, temperature, and volume) plus the amount in the cavity due to fission gas released. The gas temperature is computed as a weighted average of the pellet exterior and cladding interior surfaces, with weighting based on an approximation of the volume of gas contained between the solid surfaces. The cavity volume is computed as needed based on the evolving pellet and cladding geometry.

A variety of other material models have been implemented in BISON, often by users needing a specific model not available in the material library. These include thermal models for MOX and U3Si2 fuel, thermal and mechanical models for HT9 stainless steel cladding, irradiationinduced strain and creep models for pyrolitic carbon, and an irradiation creep model for SiC. These models are described in more detail in the BISON theory manual [13]. As mentioned above, the object-oriented architecture employed in MOOSE/BISON significantly minimizes the programming required to add new material and behavior models.

\subsection{Code dependencies: Software and Hardware}

BISON depends on several underlying software libraries. These libraries provide the finite element framework, solver technology, parallel communication, and various other functionalities. A summary of these packages is given in Table 3.1.

To make the use of these libraries consistent, the MOOSE team provides installation packages for multiple computer architectures. The packages supply pre-compiled libraries and a simple installation procedure. It is also possible to install the libraries individually if necessary.

BISON has been tested on several architectures. The bulk of the development work occurs on Apple workstations and laptops. The code is also tested on Linux machines with a variety of compilers. BISON runs on a single CPU on a laptop computer and on hundreds of CPUs on large parallel machines. 
Table 3.1: Software libraries used by BISON.

\begin{tabular}{|c|c|c|}
\hline Library & Origin & Purpose \\
\hline MOOSE[7] & Idaho National Laboratory & $\begin{array}{l}\text { Finite element framework, inte- } \\
\text { grates other packages for appli- } \\
\text { cation use }\end{array}$ \\
\hline libMesh[14] & $\begin{array}{l}\text { The University of Texas at } \\
\text { Austin }\end{array}$ & $\begin{array}{l}\text { Parallel, unstructured mesh, nu- } \\
\text { merical simulation platform }\end{array}$ \\
\hline $\operatorname{PETSc}[15]$ & Argonne National Laboratory & $\begin{array}{l}\text { Parallel linear and nonlinear } \\
\text { equation solvers }\end{array}$ \\
\hline hypre[16] & $\begin{array}{l}\text { Lawrence Livermore National } \\
\text { Laboratory }\end{array}$ & Parallel preconditioners \\
\hline $\begin{array}{l}\text { MPI (Open- } \\
\text { MPI, e.g.) }\end{array}$ & Open source consortium & $\begin{array}{l}\text { Message passing on parallel } \\
\text { computers }\end{array}$ \\
\hline
\end{tabular}




\section{Software Quality Assurance}

"Often verification and validation are parts of an overarching software quality assurance (SQA) program. SQA methods are enacted to guide the software development cycle and may include recommendations or requirements for software engineering tasks such as gathering and recording customer requirements, using revision control software, writing code, testing the software, documenting the software, and releasing the application, among others. It is common to measure the quality of the software development process by assessing how well the development team is fulfilling the requirements in each of these areas" [17].

The BISON software, along with all of the MOOSE-based software upon which it depends, is kept in a Subversion (SVN) version control repository at INL. In addition, the BISON tests and documentation are maintained in the same repository. This enables traceability of each code change. The author, date, and details of the changes are recorded with each commit to Subversion. It is also possible to retrieve a copy of the software from any point in its history. Through the use of revision control software, developers are able to maintain the current copy of BISON on their local machines and make frequent changes to BISON without fear of undoing another's changes or of making irrecoverable changes.

In addition to Subversion, the BISON team relies on software configuration management tools and practices set up for MOOSE applications. Continuous integration is accomplished through Trac. With each commit to BISON (or any MOOSE-based component), Trac automatically compiles the software across multiple computer platforms. If the compilation process is successful, the new version of the software runs the complete set of regression tests. This also happens on multiple computer platforms. This continuous integration, as it is called, helps ensure that the regression tests run correctly at all times. If a commit causes a test to fail, the failure is immediately noted through an electronic message to the developers who then correct the error.

In addition to the testing that occurs with each commit, code coverage data is also automatically produced. This information includes line and function coverage at the file, directory, and library level. The development team regularly reviews this information to ensure that the code coverage remains high.

All of this information (commit history and details, build status, test status, and code coverage) is reported through the web pages generated by Trac. Thus, the information is easily available to developers and others.

As mentioned above, the BISON team maintains its documentation in the same Subversion repository that holds the application software. The documentation includes a user manual [18], a theory manual [13], an assessment document [19], a publication list or bibliography, and a set of workshop or training presentation slides.

As BISON continues to develop and mature, it is anticipated that a formal assessment of its software quality practices will occur (using, e.g., ASME NQA-1, CMMI, or ISO 9000 standards and models). It is worth noting that MOOSE has received ASME NQA-1 certification. 


\section{Verification}

Verification, strictly speaking, is of two parts. The first is software verification, ensuring that an encoded model is mathematically correct. The second type is model verification and is the process of ensuring that the analysis model has been properly configured. The second focuses on input parameters for a given analysis, while the first focuses on computing the correct response given any input parameters. In the following discussion, the term verification refers to software verification.

Verification is the process of ensuring that a model represented in computer code calculates the correct results as defined by the mathematical definition of the model. Verification is accomplished through tests, specially designed, that exercise a particular feature of a given model. As an example, for heat conduction, we can verify that the finite element solution of a linear temperature field is in fact linear even when the mesh is composed of irregular elements. For solid mechanics, we can test that the proper stress field results when a uniform pressure is applied.

"Verification must precede Validation.... [A]ttempting to validate a model using a code that may still contain (serious) errors can lead to a false conclusion about the validity of the model" [20]. In other words, verification is a prerequisite to validation.

As a matter of engineering practice, BISON developers are responsible for developing regression tests for all of the code they develop. This helps ensure that future code changes do not break existing functionality. Some tests exercise the interaction of various models, and many others are single-feature verification tests.

By way of example, consider the process followed to introduce a new thermal conductivity model for $\mathrm{UO}_{2}$ fuel. Having identified the particular form of the model (the mathematical description), the developer can identify the inputs to the model (e.g., temperature) as well as the outputs (thermal conductivity). The developer creates a test that will exercise the new model. This test will require specific boundary conditions and perhaps other carefully controlled inputs in order to produce the exact results expected through an independent, analytic calculation.

The developer must of course also encode the relevant equations in the BISON software and compile the new code. Having done so, the developer exercises the new capability on the new test. If the computed thermal conductivity does not match the analytic expression, the developer searches for errors in the code and in the test until the discrepancy is resolved. Note that more than one verification test may be, and often is, required to give confidence that the encoded model is mathematically correct.

It should be understood that much of the underlying software in use by BISON has its own set of verification tests. In particular, verification tests concerned with the correctness of the finite element formulation are maintained at the MOOSE and libMesh levels.

BISON has many verification tests, checking solid mechanics, heat conduction, gap heat transfer, material models, mechanical contact, thermal contact, large strain capabilities, boundary conditions, plenum pressure determination, output, and many other phenomena needed for 
nuclear fuel analysis. That these tests run properly is evidence that the models have been implemented correctly.

A review of several of BISON's verification tests, along with an overview of verification in the context of nuclear fuel performance software in general and of BISON in particular, is in [17]. 


\section{Validation}

As stated in the Introduction, as BISON developers we believe that the validation effort must be coordinated between developers and users. In this vein, the BISON team has focused quite heavily on what we call an assessment effort, which is simulation of, and comparison with, well-known and well-characterized experiments from the FUMEX databases. Results from the assessment effort are presented here. With the BISON team acting as developers and users while running these FUMEX cases, the results of this effort can serve as a guide for how the BISON team may interact with users for code validation in the future.

Our goal with the assessment effort is to work toward building a suite of simulations that represent a variety of experiments in terms of material and behavior models, physics, and environments. The simulations from the assessment effort can be used as an example of building a case for validation for a particular set of conditions depending on the end use. Our hope is that the BISON assessment effort will result in the code being demonstrated as generally valid for a variety of fuel types and conditions. However, much of the responsibility for validating a specific combination of fuel-types, models, physics, and environments has to land squarely on the shoulders of users.

The following sections are organized by fuel type. As the plan evolves, individual sections can be added or modified independently. While most of our initial effort has focused on LWR fuel, we also present some preliminary assessment work on TRISO particle fuel.

In accordance with NEAM'S goals, we plan to do more assessment/validation work with metallic fuel in the future. As experimental data become available, and the long term goals of NEAMS evolve, our priorities for the assessment/validation effort will change accordingly.

\subsection{Light Water Reactor Fuel}

\subsubsection{Collaborations}

BISON developers have established several important collaborations which have strongly influenced our approach to software validation.

\section{ANATECH Corp.}

Under multiple NEAMS-funded contracts, fuel performance experts at ANATECH Corp. (developers of the FALCON [21] nuclear fuel performance code) have provided guidance on the selection and prioritization of LWR validation cases. Under a FY-14 contract, ANATECH Corp. will actively participate in validation by developing and documenting six LWR validation cases. 


\section{National Nuclear Laboratory}

The UK National Nuclear Laboratory (NNL) has considerable validation experience through validation of their ENIGMA [22] fuel performance code. As part of an informal collaborative agreement, NNL was given access to BISON and recently documented a comparison of modeling capabilities within ENIGMA and BISON [23]. In the same report they analyzed a typical AP1000 fuel rod using both codes and compared results.

Additionally, NNL has provided advice in developing BISON validation procedures. NNL's concept of specific "validation areas" was used to define similar validation areas for BISON, as detailed below. Their approach to an automated validation system is also being considered as automated validation procedures are being established for BISON.

NNL has offered INL access to a large number of non-proprietary cases in the ENIGMA validation database (233 rod irradiations including $219 \mathrm{UO}_{2}$ rods, $9 \mathrm{MOX}$ rods and 5 (U,Gd)O rods) for use in BISON validation. Planned collaborative efforts include the development of an automated approach to convert ENIGMA input data to BISON input.

\section{International Atomic Energy Agency}

Since 1981, the International Atomic Energy Agency (IAEA) has sponsored a series of Coordinated Research Projects (CRP) in the area of nuclear fuel modeling. These projects have typically lasted 3-5 years and have had broad international participation. The objectives of the projects have been to assess the maturity and predictive capability of fuel performance codes, support interaction and information exchange between countries with code development and application needs, build a database of well-defined experiments suitable for code validation, transfer a mature fuel modeling code to developing countries, and provide guidelines for code quality assurance and code application to fuel licensing. The fourth and latest of these projects, known as FUMEX-III (FUel Modeling at EXtended Burnup-III) [24] began in 2008 and ended in December of 2011.

INL participated in FUMEX-III using an early version of BISON (ref). Participation resulted in access to a large amount of well documented and trusted experimental data from both the FUMEX-II [25] and III CRP's. As described below, the majority of BISON validation cases both completed and planned are based on experiments from the FUMEX exercises.

The next IAEA CRP related to LWR fuel performance modeling is scheduled to begin in November 2014 and will be known as Fuel Modeling Under Accident Conditions (FUMAC). INL was involved in the preliminary planning of this CRP and will be an active participant. This exercise will provide both experimental data and collaboration with international experts as BISON validation efforts are extended into accident regimes.

\section{OCED Halden Reactor Project}

The INL has enjoyed a long association with the OECD Halden Reactor Project. This interaction has helped us understand and interpret several Halden experiments included in the current BISON validation database. In particular, BISON was used to analyze a unique 3D Halden experiment involving eccentrically located fuel [26]. INL has also recently used BISON to aid in the preliminary design of a planned Halden experiment, and further collaboration on future 
experiment designs is anticipated. It is expected that this relationship will continue to help us validate BISON well into the future.

\section{European Commission - Institute for Transuranium Elements and Politecnico di Milano}

The European Commission's JRC Institute for Transuranium Elements (ITU, Germany) and the Politecnico di Milano (POLIMI, Italy) have long been involved in fuel modeling research with the development and validation of the TRANSURANUS code [27, 28], which is today the most widely used fuel performance code in the European Union. Very recently, an INL-ITUPOLIMI R\&D collaboration has been established. The collaboration will focus initially on the development and validation of improved models of inert gas ( $\mathrm{Xe}, \mathrm{Kr}, \mathrm{He}$ ) behavior in oxide fuel, with special reference to accident regimes. The developed models will be implemented in both BISON and TRANSURANUS, and a benchmark between the two codes is foreseen. The collaboration is expected to enhance the BISON development and validation processes through sharing of code improvements and knowledge - and through researchers' mobility - between the involved institutions.

\subsubsection{Validation Approach}

Our general approach to evaluating BISON as a predictive tool is to create and run BISON simulations of well known and documented experiments, then compare BISON calculations to the recorded experiment measurements. This process continues by adding more so-called assessment or validation cases and modifying the code to improve the agreement between simulation and experiment.

The early part of this assessment effort has focused primarily on LWR fuel experiments from the FUMEX database. The cases analyzed so far are documented in detail and made available to all BISON users via the SVN repository. Each document contains an overview of the specific experiment, specifications of geometry and materials, and simplifications and assumptions used for simulation. Each document also includes boundary conditions used and specific BISON models used for simulation. The results are compared to experimental data and, in some instances, results from simulations by other codes. A section at the end of each document is reserved for discussion on the simulation, what issues were encountered, and how those issues were resolved along with assumptions and simplifications made to data used as input. A summary document is released at the end of each fiscal year; the latest document was released with the release of BISON 1.0 [19].

BISON and its supporting software is modified frequently. As such, running all our assessment cases daily gives us the ability to see how modifications have affected the assessment calculations. Structured after our regression system (see Verification section), an automated system runs each of our assessment cases every 24 hours.

As this automated system evolves, each case will be compared to a case-dependent gold standard. Statistical analysis and plotting will permit an evaluation of code validation status and an indication of whether code changes have adversely affected simulation/experiment comparisons. Systematic comparisons to experimental data will be made in each of the validation areas. 
For BISON to reach the status of being generally considered as a validated code and useful predictive tool, it is essential that the simulation-to-experiment comparison database expands and improves. As each new case is analyzed, it will be added to the set that are run each day. In addition, the validation system will be available to users to permit addition of their own cases and allow them to analyze cases according to their specific validation requirements.

\subsubsection{Validation Areas}

Nuclear fuel behavior is highly complex with critical fuel life limiting parameters, such as peak fuel temperature and rod internal pressure, dependent on a wide variety of physical phenomena. Validation of LWR fuel behavior is typically done by identifying several validation areas [23] generally defined by the availability of experimental data. Although still being formulated, the following areas have been defined for LWR fuel:

1. Beginning-of-life fuel centerline temperature (BOL FCT)

2. Ramp-test fuel centerline temperature (Ramp FCT)

3. Through-life fuel centerline temperature (TL FCT)

4. Through-life rod internal pressure (TL Press)

5. End-of-life fission gas release (EOL FGR)

6. End-of-life fuel grain size (EOL Grain Size)

7. End-of-life rod free volume (EOL Volume)

8. End-of-life fuel pellet density (EOL Density)

9. End-of-cycle cladding diameter change (EOC Cladding Dia)

10. End-of-cycle cladding waterside oxide thickness (EOC Oxide)

11. Fuel stack elongation (Fuel Elong)

12. Rod length change (Cladding Elong)

\subsubsection{Current Status}

To date, 24 validation cases have been considered, as summarized in Table 6.1. Indicated in the table are the measured quantities for comparison, namely fuel centerline temperature (FCT) at beginning of life (BOL), throughout life (TL) and during power ramps (Ramps), fission gas release (FGR), cladding outer diameter following pellet cladding mechanical interaction (Cladding Dia), and cladding elongation (Cladding Elong). Many of these assessment cases were chosen due to participation in the IAEA sponsored FUMEX Coordinated Research Projects and are priority cases from either FUMEX-II [25] or FUMEX-III [29]. Other cases were chosen based on recommendations from nuclear fuel experts. Not all of the validation areas listed in section 6.1.3 have been considered for these cases, but will be included in future work. For details concerning completed assessment cases see [19, 30]. 
Table 6.1: Summary table of BISON LWR assessment cases.

\begin{tabular}{l||l|c|c|c|c|c|c} 
Experiment & Rod & $\begin{array}{c}\text { BOL } \\
\text { FCT }\end{array}$ & $\begin{array}{c}\text { Ramp } \\
\text { FCT }\end{array}$ & $\begin{array}{c}\text { TL } \\
\text { FCT }\end{array}$ & $\begin{array}{c}\text { EOL } \\
\text { FGR }\end{array}$ & $\begin{array}{c}\text { EOC } \\
\text { Cladding } \\
\text { Dia }\end{array}$ & $\begin{array}{c}\text { Cladding } \\
\text { Elong }\end{array}$ \\
\hline IFA-431 & $1,2,3$ & $\mathrm{X}$ & & & & & \\
\hline IFA-432 & $1,2,3$ & $\mathrm{X}$ & & & & & \\
\hline IFA-513 & 1,6 & $\mathrm{X}$ & & $\mathrm{X}$ & & & \\
\hline IFA-515.10 & A1 & $\mathrm{X}$ & & & & & \\
\hline IFA-597.3 & 7,8 & & $\mathrm{X}$ & & & & $\mathrm{X}$ \\
\hline Ris $\varnothing-3$ & AN3,AN4 & & $\mathrm{X}$ & & $\mathrm{X}$ & & \\
\hline AREVA & & & & & $\mathrm{X}$ & & \\
\hline FUMEX-II & $27(1,2 \mathrm{a}-\mathrm{d})$ & & & & $\mathrm{X}$ & & \\
\hline Ris $\varnothing-3$ & GE7 & & & & $\mathrm{X}$ & & \\
\hline OSIRIS & J12 & & & & & $\mathrm{X}$ & \\
\hline REGATE & & & & & $\mathrm{X}$ & $\mathrm{X}$ & \\
\hline PWR 16x16 & TSQ002 & & & $\mathrm{X}$ & $\mathrm{X}$ & $\mathrm{X}$ & \\
\hline IFA-431 (3D) & 4 & $\mathrm{X}$ & & & & &
\end{tabular}

\subsubsection{Planned Work}

Prioritized tables of planned validation cases are shown in Tables 6.2 and Table 6.3. An effort has been made to cover all validation areas specified in section 6.1.3, however, not all experiments have been thoroughly researched to date. With better understanding of available experimental data, the tables will become more fully populated.

The tabulated cases were prioritized by FUMEX-II priority cases first, followed by FUMEXIII cases, concluding with the rest of the FUMEX-II cases. Not all FUMEX cases are listed, only those that can be considered using the current version of BISON. 
Table 6.2: Prioritized summary table for the upcoming validation cases for validation areas 1-6 specified in section 6.1.3.

\begin{tabular}{|c|c|c|c|c|c|c|c|}
\hline Experiment & Rod & $\begin{array}{l}\text { BOL } \\
\text { FCT }\end{array}$ & $\begin{array}{c}\text { Ramp } \\
\text { FCT }\end{array}$ & $\begin{array}{c}\mathrm{TL} \\
\mathrm{FCT} \\
\end{array}$ & $\begin{array}{c}\text { TL } \\
\text { Press } \\
\end{array}$ & $\begin{array}{l}\text { EOL } \\
\text { FGR } \\
\end{array}$ & $\begin{array}{c}\text { EOL } \\
\text { Grain } \\
\text { Size }\end{array}$ \\
\hline IFA-534.14 & 18 & & & & $X$ & $\mathrm{X}$ & $X$ \\
\hline IFA-534.14 & 19 & & & & $\mathrm{X}$ & $X$ & $X$ \\
\hline HBEP & BK363 & & & & & $X$ & $X$ \\
\hline HBEP & BK365 & & & & & $\mathrm{X}$ & $X$ \\
\hline HBEP & BK370 & & & & & $X$ & $X$ \\
\hline R. E. Ginna & & & & & $X$ & $X$ & \\
\hline OSIRIS & H09 & & & & & $X$ & \\
\hline Ris $\emptyset-3$ & II5 & & $X$ & & $\mathrm{X}$ & $X$ & \\
\hline Ris $\varnothing-3$ & III & & $\mathrm{X}$ & & $\mathrm{X}$ & $X$ & $X$ \\
\hline Ris $\varnothing-3$ & GE-m & & $X$ & & $X$ & $X$ & $X$ \\
\hline IFA-535.5/6 & 809 & & & & $\mathrm{X}$ & $\mathrm{X}$ & \\
\hline IFA-535.5/6 & 810 & & & & $\mathrm{X}$ & $\mathrm{X}$ & \\
\hline IFA-535.5/6 & 811 & & & & $\mathrm{X}$ & $X$ & \\
\hline IFA-535.5/6 & 812 & & & & $X$ & $X$ & \\
\hline IFA-519.8/9 & DC & & $X$ & & $X$ & & \\
\hline IFA-519.8/9 & DK & & $\mathrm{X}$ & & $\mathrm{X}$ & & \\
\hline \multicolumn{8}{|l|}{ INTER-RAMP } \\
\hline SUPER-RAMP & & & & & & & $\mathrm{X}$ \\
\hline Ris $\varnothing-3$ & AN2 & & & & & $X$ & $X$ \\
\hline TRIBULATION & $\mathrm{BN} 1 / 3$ & & & & & $\mathrm{X}$ & \\
\hline TRIBULATION & $\mathrm{BN} 1 / 4$ & & & & & $X$ & \\
\hline TRIBULATION & BN1/15 & & & & & $X$ & \\
\hline IFA-507 & TF3 & $\mathrm{X}$ & $\mathrm{X}$ & $X$ & & & \\
\hline IFA-507 & TF5 & $\mathrm{X}$ & $\mathrm{X}$ & $X$ & & & \\
\hline HATAC & & & & & & $X$ & \\
\hline
\end{tabular}


Table 6.3: Prioritized summary table for the upcoming validation cases for validation areas 7-12 specified in section 6.1.3.

\begin{tabular}{|c|c|c|c|c|c|c|c|}
\hline Experiment & Rod & $\begin{array}{c}\text { EOL } \\
\text { Volume }\end{array}$ & $\begin{array}{c}\text { EOL } \\
\text { Density }\end{array}$ & $\begin{array}{c}\text { EOC } \\
\text { Cladding } \\
\text { Dia }\end{array}$ & $\begin{array}{c}\text { EOC } \\
\text { Oxide }\end{array}$ & $\begin{array}{c}\text { Fuel } \\
\text { Elong }\end{array}$ & $\begin{array}{c}\text { Cladding } \\
\text { Elong }\end{array}$ \\
\hline IFA-534.14 & 18 & $X$ & & & $\mathrm{X}$ & & \\
\hline IFA-534.14 & 19 & $\mathrm{X}$ & & & $\mathrm{X}$ & & \\
\hline HBEP & BK363 & & & & $\mathrm{X}$ & & \\
\hline HBEP & BK365 & & & & $\mathrm{X}$ & & \\
\hline HBEP & BK370 & & & & $\mathrm{X}$ & & \\
\hline R. E. Ginna & & $\mathrm{X}$ & & $\mathrm{X}$ & & $X$ & $\mathrm{X}$ \\
\hline OSIRIS & H09 & $\mathrm{X}$ & $\mathrm{X}$ & $\mathrm{X}$ & $\mathrm{X}$ & $\mathrm{X}$ & $\mathrm{X}$ \\
\hline Ris $\varnothing-3$ & II5 & $\mathrm{X}$ & & $\mathrm{X}$ & $\mathrm{X}$ & & $\mathrm{X}$ \\
\hline Ris $\varnothing-3$ & II3 & $\mathrm{X}$ & & $\mathrm{X}$ & $\mathrm{X}$ & & \\
\hline $\operatorname{Ris} \varnothing-3$ & GE-m & & & $\mathrm{X}$ & $\mathrm{X}$ & & \\
\hline IFA-535.5/6 & 809 & $\mathrm{X}$ & & & & & $\mathrm{X}$ \\
\hline IFA-535.5/6 & 810 & $\mathrm{X}$ & & & & & $\mathrm{X}$ \\
\hline IFA-535.5/6 & 811 & $\mathrm{X}$ & & & & & $\mathrm{X}$ \\
\hline IFA-535.5/6 & 812 & $X$ & & & & & $X$ \\
\hline IFA-519.8/9 & DC & $\mathrm{X}$ & & & & & \\
\hline IFA-519.8/9 & DK & $\mathrm{X}$ & & & & & \\
\hline INTER-RAMP & & & & & & $\mathrm{X}$ & $\mathrm{X}$ \\
\hline SUPER-RAMP & & & & & $\mathrm{X}$ & & \\
\hline Ris $\varnothing-3$ & AN2 & $\mathrm{X}$ & & & $\mathrm{X}$ & & \\
\hline TRIBULATION & $\mathrm{BN} 1 / 3$ & $\mathrm{X}$ & & $\mathrm{X}$ & & $\mathrm{X}$ & $\mathrm{X}$ \\
\hline TRIBULATION & $\mathrm{BN} 1 / 4$ & $\mathrm{X}$ & & $\mathrm{X}$ & & $\mathrm{X}$ & $X$ \\
\hline TRIBULATION & BN1/15 & $\mathrm{X}$ & & $\mathrm{X}$ & & $\mathrm{X}$ & $\mathrm{X}$ \\
\hline IFA-507 & TF3 & & & & & & \\
\hline IFA-507 & TF5 & & & & & & \\
\hline HATAC & & $\mathrm{X}$ & & $\mathrm{X}$ & & & \\
\hline
\end{tabular}




\subsection{TRISO Particle Fuel}

The major development and $\mathrm{V} \& \mathrm{~V}$ focus for BISON to date has been oxide fuel in light water reactors. A smaller but significant development effort has resulted in extension of this capability to include oxide fuel in a TRISO-coated particle fuel configuration. New material models required for this fuel type were verified as part of the development process, as described above.

Preliminary code assessment has been accomplished by comparing BISON results to predictions from other well-known and validated particle fuel codes. Although this is not validation in the strictest sense (since it does not involve comparison to experimental data) it is nonetheless an important and useful exercise, particularly in cases where experimental data is limited.

\subsubsection{Code comparisons}

As part of an International Atomic Energy Agency (IAEA) Coordinated Research Program (CRP-6) on HTGR reactor fuel technology, a set of benchmarking activities were developed to compare fuel performance codes under normal operation and operational transients [31]. Sixteen benchmark cases were identified, ranging in complexity from a simple fuel kernel having a single elastic coating layer, to realistic TRISO-coated particles under a variety of irradiation conditions. In each case, the particle geometry, constitutive relations, material properties and operating conditions were carefully prescribed to minimize differences between the various code predictions; details are given in [31]. As a preliminary code assessment exercise, BISON has been applied to 13 of the 16 benchmark cases. This exercise is summarized in Table 6.4.

Table 6.4: IAEA CRP-6 benchmark cases considered in the BISON coated-particle fuel verification exercise. HFR-K3 and HFR-P4 are German pebble and fuel element experiments, respectively.

\begin{tabular}{lll}
\hline Case & Geometry & Description \\
\hline 1 & SiC layer & Elastic only \\
2 & IPyC layer & Elastic only \\
3 & IPyC/SiC & Elastic with no fluence \\
$4 \mathrm{a}$ & IPyC/SiC & Swelling and no creep \\
$4 \mathrm{~b}$ & IPyC/SiC & Creep and no swelling \\
$4 \mathrm{c}$ & IPyC/SiC & Creep and swelling \\
$4 \mathrm{~d}$ & IPyC/SiC & Creep- and fluence-dependent swelling \\
5 & TRISO & $350 \mu$ m kernel, real conditions \\
6 & TRISO & $500 \mu$ m kernel, real conditions \\
7 & TRISO & Same as 6 with high BAF PyC \\
8 & TRISO & Same as 6 with cyclic temperature \\
10 & HFR-K3 & $10 \%$ FIMA, $5.3 \times 10^{-25} \mathrm{n} / \mathrm{m}^{2}$ fluence \\
11 & HFR-P4 & $14 \%$ FIMA, $7.2 \times 10^{-25} \mathrm{n} / \mathrm{m}^{2}$ fluence \\
\hline
\end{tabular}


Cases 1 to 3 were limited to single and double coating layers and tested simple elastic thermomechanical behavior against analytical solutions. A comparison of the analytical and BISON numerical solutions for the maximum tangential stress, which occurs at the inner surface of the various layers, showed excellent agreement.

Cases $4 \mathrm{a}$ to $4 \mathrm{~d}$ included both IPyC and $\mathrm{SiC}$ layers and investigated pyrolytic carbon layer behavior under a variety of conditions. Cases 5 to 8 considered a single TRISO particle with more complexity added with each subsequent case. For cases 1 to $4 d$, the internal gas pressure was fixed at $25 \mathrm{MPa}$ while cases 5 to 8 included a linear pressure ramp. The particle temperature was held uniform at $1273 \mathrm{~K}$ for cases 1 to 7 , but for case 8 was cycled ten times between 873 and $1273 \mathrm{~K}$, characteristic of fuel in a pebble bed reactor.

BISON solutions of the tangential stress at the inner surface of both the IPyC and SiC layers were compared to the range of solutions from eight coated-particle fuel codes included in the CRP-6 exercise (see [31]) and were always within the range of values computed by the other codes. As an example, Fig. 6.1 compares solutions for case 8, which involved a cyclic particle temperature, during the full irradiation history. In this figure, BISON solutions of the tangential stress at the inner wall of the IPyC and SiC layers are compared to solutions from three codes from the CRP-6 exercise, namely PARFUME [32], ATLAS [33] and STRESS3 [34]. For the IPyC layer, the four solutions essentially overlay each other during the entire irradiation period. In the $\mathrm{SiC}$ layer, the four solutions are quite similar but some differences are evident, particularly for the first four temperature cycles. The BISON solution falls roughly midway between the PARFUME and STRESS3 solutions and is essentially identical to the ATLAS solution.

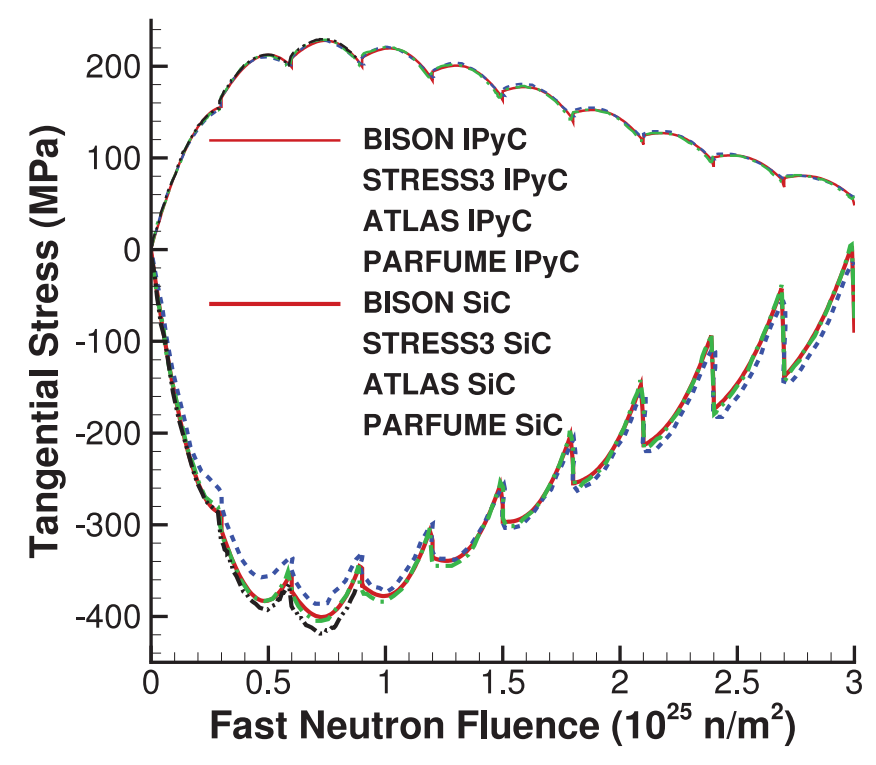

Figure 6.1: Code comparison for case 8, which included a ten cycle temperature history. Plotted is the tangential stress at the inner wall of the IPyC and $\mathrm{SiC}$ layers. 
Cases 9 to 13 in CRP-6 were more complicated benchmarks based on past or planned experiments with TRISO-coated particles. The two cases considered to date (10 and 11) were based on German fuel from pebble and fuel element experiments. Again, details are provided in [31]. Although material properties and constitutive relations were prescribed for these cases, they differed from cases 1 to 8 in two important ways: (1) the internal pressure was not fixed but instead determined by fission gas release and $\mathrm{CO}$ production and (2) the particle size was prescribed as a population (mean value and standard deviation) rather than a single value. BISON was compared to three codes from the CRP-6 exercise and substantial differences exist in the solutions, particularly for the gas pressure. The BISON solution histories, however, compare well to the range of solutions given by the three well-established codes chosen for comparison. As stated in [31], the differences between various code predictions can be largely attributed to the models used to calculate fission gas release and $\mathrm{CO}$ production in the kernel. These two cases clearly point out the limitation of code comparisons in the absence of experimental data.

Complete details on this preliminary assessment of BISON for TRISO-coated particle fuel are included in reference [2].

\subsubsection{Future work}

There are currently no plans within NEAMS to extend the BISON validation base for particle fuel. This is an area where user involvement will be essential. 


\subsection{Metallic Fuel}

\subsubsection{Collaborations}

NEAMS and Fuel Cycle Research and Development (FCRD) leadership has indicated that future development effort should include metallic fuel for sodium fast reactors. The BISON team has had recent interaction with the Nuclear Energy Agency (NEA), which is part of Organization for Economic Co-operation and Development (OECD). A group within NEA called the Working Party on Scientific Issues of the Fuel Cycle plan to share experimental data from a variety of test programs in different countries and compare those experiments to calculations from participants' fuel performance simulation software. These experiments focus on metallic and MOX fuel. The INL has an opportunity to engage in this effort. Future verification, assessment, and validation efforts could be planned based on working with this group. 


\section{Uncertainty Quantification}

Fuel performance analysis involves uncertainties associated with fuel fabrication/characterization and irradiation parameters, material properties, and modeling assumptions. In order to assess the technological effect of uncertainties and determine the safety criteria, uncertainty quantification and sensitivity analysis are performed.

\subsection{Accomplished results}

Work has been done with BISON to investigate the role of uncertainties in fuel performance analyses, focusing initially on the uncertainties associated with fission gas behavior modeling [35]. Indeed, the confidence in fuel thermomechanics calculations by means of fuel performance codes significantly depends on the confidence in this aspect of fuel analysis. Gaseous fuel swelling promotes pellet-cladding mechanical interaction (PCMI), and the concomitant fission gas release (FGR) to the fuel rod free volume increases the rod internal pressure, both processes affecting the mechanical behavior of the cladding. Moreover, gas retention and release affect the fuel thermal conductivity and the thermal conductance of the fuel-cladding gap, respectively, and consequently the temperature distribution in the fuel pellet [36, 37].

Through the integration of BISON with the DAKOTA framework [38], a systematic sensitivity analysis of fuel calculations to selected uncertain parameters of the fission gas behavior model (Section 3.1) was carried out. The parameters were varied within ranges representative of the relative uncertainties and consistent with the information in the open literature.

Based on $\mathrm{UO}_{2}$ single-pellet simulations covering a broad range of LWR operating conditions, the variability of FGR in response to the considered uncertainties was assessed. The results led to a quantitative assessment of the uncertainty in the calculated FGR with the parameter characterization available at present. In particular, a FGR variability of a factor of 2 and higher, depending on irradiation conditions, was demonstrated. Moreover, the effect of fission gas behavior uncertainties on cladding diameter predictions for a fuel rod irradiation experiment involving PCMI $[39,40]$ was assessed. The results are presented in Fig. 7.1. The significant impact of the considered uncertainties on the calculated fuel rod behavior in terms of cladding diametral strain is evident.

The uncertainties in the intra-granular gas atom diffusion coefficient, resolution parameter, calculated fuel temperature and grain size were identified as most contributing to fission gas release and swelling uncertainties. A better characterization of such parameters through experimental and theoretical research may significantly reduce the uncertainty in fission gas behavior calculations and in the multiple related aspects of fuel performance analysis. 


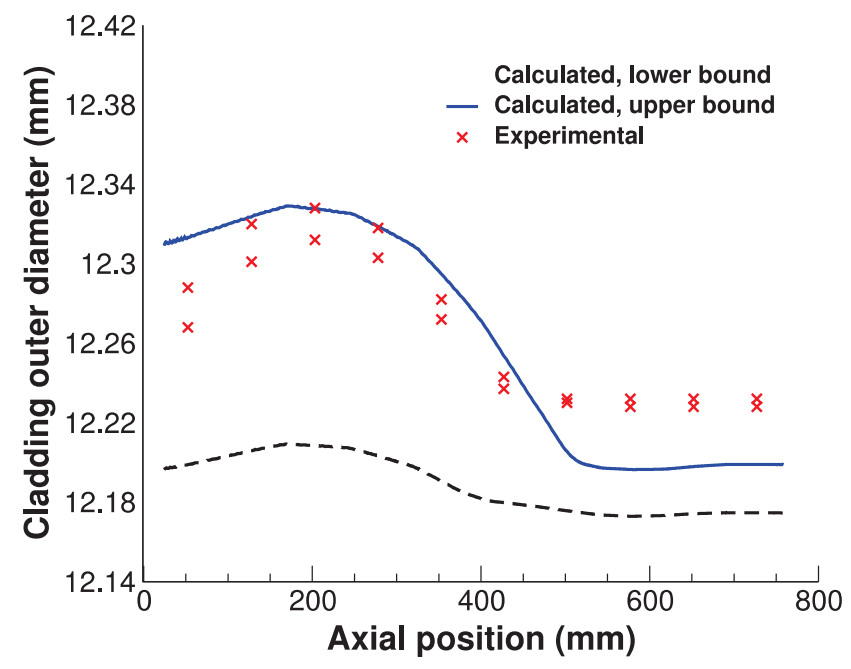

Figure 7.1: Cladding outer diameter as a function of axial position at the end of a fuel rod irradiation. The fabricated cladding outer diameter is $12.26 \mathrm{~mm}$.

\subsection{Future work}

Work is planned to investigate the role of uncertainties associated with the heat transfer in the fuel-cladding gap. Initial sensitivity studies have been performed on parameters governing the heat transfer model such as the roughness coefficient, the jump coefficient, and the fuel thermal conductivity. Additional studies will be performed which will make use of uncertainty quantification techniques such as Latin hypercube sampling. Given distributions on selected model parameters, distributions on the output (e.g., fuel centerline temperature) will be sought. The study will focus initially on early fuel life and will be subsequently extended to end-of-life fuel behavior. Reference will be made to the Halden IFA series of irradiation experiments [25]. 


\section{Supporting Documents}

The following documents give an overview of BISON capabilities, information related to software quality practices of BISON development, and details concerning verification and validation of the software.

- BISON Overview [1]

- BISON for TRISO fuel [2]

- BISON Verification [17]

- BISON Theory Manual [13]

- BISON Users Manual [18]

- BISON Assessment Document [19] 


\section{Bibliography}

[1] R. L. Williamson, J. D. Hales, S. R. Novascone, M. R. Tonks, D. R. Gaston, C. J. Permann, D. Andrs, and R. C. Martineau. Multidimensional multiphysics simulation of nuclear fuel behavior. J. Nucl. Mater., 423:149-163, 2012.

[2] J. D. Hales, R. L. Williamson, S. R. Novascone, D. M. Perez, B. W. Spencer, and G. Pastore. Multidimensional multiphysics simulation of TRISO particle fuel. J. Nucl. Mater, 443:531-543, 2013.

[3] Pavel Medvedev. Fuel performance modeling results for representative FCRD irradiation experiments: Projected deformation in the annular AFC-3A U-10Zr fuel pins and comparison to alternative designs. Technical Report INL/EXT-12-27183 Revision 1, Idaho National Laboratory, 2012.

[4] N. N. Carlson, C. Unal, and J. D. Galloway. Formulation of the constituent distribution model implemented into the BISON framework for the analysis of performance of metallic fuels with some initial simulation results. Technical Report LA-UR-13-26824, Los Alamos National Laboratory, 2013.

[5] Pavel Medvedev. Summary report on the fuel performance modeling of the AFC-2A, 2B irradiation experiments. Technical Report INL/EXT-13-30006, Idaho National Laboratory, 2013.

[6] K. E. Metzger, T. W. Knight, and R. L. Williamson. Model of $\mathrm{U}_{3} \mathrm{Si}_{2}$ fuel system using BISON fuel code. In Proceedings of the International Congress on Advances in Nuclear Power Plants - ICAPP 2014, Charlotte, NC, April 6-9 2014.

[7] D. Gaston, C. Newman, G. Hansen, and D. Lebrun-Grandié. MOOSE: A parallel computational framework for coupled systems of nonlinear equations. Nucl. Eng. Design, 239:1768-1778, 2009.

[8] D. A. Knoll and D. E. Keyes. Jacobian-free Newton-Krylov methods: a survey of approaches and applications. J. Comput. Phys., 193(2):357-397, 2004.

[9] J. D. Hales, S. R. Novascone, R. L. Williamson, D. R. Gaston, and M. R. Tonks. Solving nonlinear solid mechanics problems with the Jacobian-free Newton Krylov method. CMES: Comput. Model. Eng. Sci., 84(2):123-154, 2012.

[10] G. Pastore, L. Luzzi, V. Di Marcello, and P. Van Uffelen. Physics-based modelling of fission gas swelling and release in $\mathrm{UO}_{2}$ applied to integral fuel rod analysis. Nuclear Engineering and Design, 256:75-86, 2013. 
[11] A. M. Ross and R. L. Stoute. Heat transfer coefficient between $\mathrm{UO}_{2}$ and Zircaloy-2. Technical Report AECL-1552, Atomic Energy of Canada Limited, 1962.

[12] J. D. Hales, D. Andrs, and D. R. Gaston. Algorithms for thermal and mechanical contact in nuclear fuel performance analysis. In Proceedings of the International Conference on Mathematics and Computational Methods Applied to Nuclear Science and Engineering, Sun Valley, Idaho, May 5-9, 2013.

[13] J. D. Hales, S. R. Novascone, G. Pastore, D. M. Perez, B. W. Spencer, and R. L. Williamson. BISON theory manual: The equations behind nuclear fuel analysis. Technical Report INL/EXT-13-29930, Idaho National Laboratory, September 2013.

[14] Benjamin S. Kirk, John W. Peterson, Roy H. Stogner, and Graham F. Carey. libMesh: A C++ library for parallel adaptive mesh refinement/coarsening simulations. Engineering with Computers, 22(3-4):237-254, January 2006.

[15] Portable, extensible toolkit for scientific computation. http://www.mcs.anl.gov/petsc/. Retrieved April 25, 2012.

[16] Robert D. Falgout and Ulrike Meier Yang. HYPRE: A library of high performance preconditioners. In International Conference on Computational Science (3), pages 632-641, 2002.

[17] J. D. Hales, S. R. Novascone, B. W. Spencer, R. L. Williamson, G. Pastore, and D. M. Perez. Verification of the BISON fuel performance code. Ann. Nuclear Energy, 71:81-90, September 2014.

[18] J. D. Hales, S. R. Novascone, G. Pastore, D. M. Perez, B. W. Spencer, and R. L. Williamson. BISON users manual. Technical Report INL/MIS-13-30307, Idaho National Laboratory, September 2013.

[19] D. M. Perez, R. L. Williamson, S. R. Novascone, G. Pastore, J. D. Hales, and B. W. Spencer. Assessment of BISON: A nuclear fuel performance analysis code. Technical Report INL/MIS-13-30314, Idaho National Laboratory, September 2013.

[20] L. E. Schwer. An overview of the PTC 60/V\&V 10: Guide for verification and validation in computational solid mechanics. Engrg. Comput., 23:245-252, 2007.

[21] Y. Rashid, R. Dunham, and R. Montgomery. Fuel analysis and licensing code: FALCON MOD01. Technical Report EPRI 1011308, Electric Power Research Institute, December 2004.

[22] Glyn Rossiter. Development of the ENIGMA fuel performance code for whole core analysis and dry storage assessments. Nuclear Engineering and Technology, 43(6):489-498, 2011.

[23] Glyn Rossiter. Comparison of the BISON and ENIGMA fuel performance codes. Technical Report IP20084.433/06/10/01, National Nuclear Laboratory, 2013. 
[24] E. Sartori J. Killeen and T. Tverberg. FUMEX-III: A New IAEA Coordinated Research Project on Fuel Modeling at Extended Burnup. In Proceedings of Top Fuel Paper 2176, Paris, France, Sep. 2009.

[25] IAEA. Fuel Modelling at Extened Burnup (FUMEX-II): Report of a Coordinated Research Project 2002-2007. Technical Report IAEA-TECDOC-1687, International Atomic Energy Agency, 2002-2007.

[26] J. D. Hales, D. M. Perez, R. L. Williamson, S. R. Novascone, B. W. Spencer, and R. C. Martineau. Validation of the BISON 3D fuel performance code: Temperature comparisons for concentrically and eccentrically located fuel pellets. In Enlarged Halden Programme Group Meeting: Proceedings of the Fuels and Materials Sessions, volume HPR378, Storefjell Resort Hotel, Norway, March 10-15 2013. OECD Halden Reactor Project.

[27] K. Lassmann and H. Blank. Modelling of fuel rod behavior and recent advances of the TRANSURANUS code. Nucl. Engrg. Design, 106:291-313, 1988.

[28] K. Lassmann. TRANSURANUS: a fuel rod analysis code ready for use. J. Nucl. Mater., 188:295-302, 1992.

[29] IAEA. Improvement of Computer Codes Used for Fuel Behaviour Simulation (FUMEXIII): Report of a Coordinated Research Project 2008-2012. Technical Report IAEATECDOC-1697, International Atomic Energy Agency, 2008-2012.

[30] D. M. Perez, R. L. Williamson, S. R. Novascone, T. K. Larson, J. D. Hales, B. W. Spencer, and G. Pastore. An evaluation of the nuclear fuel performance code BISON. In Proceedings of the International Conference on Mathematics and Computational Methods Applied to Nuclear Science and Engineering, Sun Valley, Idaho, May 5-9, 2013.

[31] IAEA. Advances in high temperature gas cooled reactor fuel technology. Technical Report IAEA-TECDOC-1674, International Atomic Energy Agency, 2012.

[32] G. K. Miller, D. A. Petti, J. T. Maki, and D. L. Knudsen. PARFUME theory and model basis report. Technical Report INL/EXT-08-14497, Idaho National Laboratory, 2009.

[33] M. Phelip, F. Michel, M. Pelletier, G. Degeneve, and P. Guillermier. The ATLAS HTR fuel simulation code objectives, description and first results. In 2nd International Topical Meeting on High Temperature Reactor Technology, pages 1-10, Beijing, China, September 2004.

[34] D. G. Martin. Considerations pertaining to the achievement of high burn-ups in htr fuel. Nuclear Engineering and Design, 213:241-258, 2002.

[35] G. Pastore, L. P. Swiler, J. D. Hales, D. M. Perez, B. W. Spencer, L. Luzzi, P. Van Uffelen, and R. L. Williamson. Uncertainty and sensitivity analysis of fission gas behavior in engineering-scale fuel modeling. Journal of Nuclear Materials, submitted, 2014.

[36] D. R. Olander. Fundamental aspects of nuclear reactor fuel elements. Technical Information Center, Energy Research and Development Administration, 1976. 
[37] P. Van Uffelen, R. J. M. Konings, C. Vitanza, and J. Tulenko. Analysis of reactor fuel rod behavior. In D. G. Cacuci, editor, Handbook of Nuclear Engineering, volume 13, pages 1519-1627. Springer Science + Business Media, LLC., New York, NY, USA, 2010.

[38] B. M. Adams, K. R. Dalbey, M. S. Eldred, L. P. Swiler, W. J. Bohnhoff, J. P. Eddy, D. M. Vigil, P. D. Hough, and S. Lefantzi. DAKOTA: A multilevel parallel object-oriented framework for design optimization, parameter estimation, uncertainty quantification, and sensitivity analysis. Version 5.2 user's manual. Technical Report SAND2010-2183, 2011.

[39] The Third Risø Fission Gas Project: Bump Test GE7 (ZX115). Technical Report RISØFGP3-GE7, 1990.

[40] E. Sartori, J. Killeen, and J. A. Turnbull. International Fuel Performance Experiments (IFPE) Database. OECD-NEA, 2010, available at http://www.oecdnea.org/science/fuel/ifpelst.html. 